\title{
SIMULATION AND PERFORMANCE ANALYSIS OF BLAST STRUCTURE IN MIMO ENVIRONMENT
}

\author{
Nirali A. Kotak ${ }^{1}$, Ved Vyas Dwivedi ${ }^{2}$ \\ ${ }^{1}$ Research Scholar, Department of Electronics \& comm., J.J.T. University, Rajasthan, India \\ ${ }^{2}$ Guide and, Pro-vice Chancellor, C.U.Shah University, Gujarat, India
}

\begin{abstract}
This paper presents the modeling and simulation of the most innovative MIMO-VBLAST technique. Wireless communication has suffered from the fading problem ever since its first appearance in 1897, when Guglielmo Marconi transmitted a wireless signal to a ship in the English Channel. The following century witnessed the remarkable development of wireless communication, especially in the last decade. The capacity of traditional communication systems along with 1xI antenna system would be very low because of multi path propagation within radio channels. The multi-path signals add up constructively or destructively at the receiver antenna to give a fluctuating signal, which can vary widely in amplitude and phase. When the amplitude of the signal experiences a low value it is termed fading and the capability of the wireless channel is severely limited. Several efforts into this direction have been carried out to make the radio channel more efficient and this has got remarkable progress. Efficient techniques, such as frequency reuse and OFDM, have been invented to increase the bandwidth efficiency; on the other hand, advances in coding techniques, such as Reed Solemn codes, Convolution codes and low density parity check (LDPC) codes make it possible to almost reach Shannon capacity the theoretical performance limit of the channel. However, the development of the techniques for a single channel has yet to catch up with the increasing demand for the capacity The most promising solution to combat this effect is to have antenna diversity scheme which can be implemented with many of ways such as BLAST structure, STBC coding technique, etc. With the implementation of antenna diversity i.e. MIMO, the capacity of the communication system as well as channel will increase in terms of improved error rate performance. Also with the implementation of BLAST structure the data rate of the communication can be enhanced at the same amount of bandwidth. Ultimately this paper concludes effect of MIMO-BLAST to provide array gain i.e high data rate for modern wireless scenario.
\end{abstract}

Keywords: MIMO System, V-BLAST structure, STBC, AWGN Channel, BER \& SNR. $* * *$

\section{INTRODUCTION}

To achieve the maximum advantage of antenna diversity technique, MIMO is the best option where in the idea is lying within the fact that the multiple no. of transmitter and receiver antennas should be placed enough far apart so as to have at least one single path which incurs the least fading.

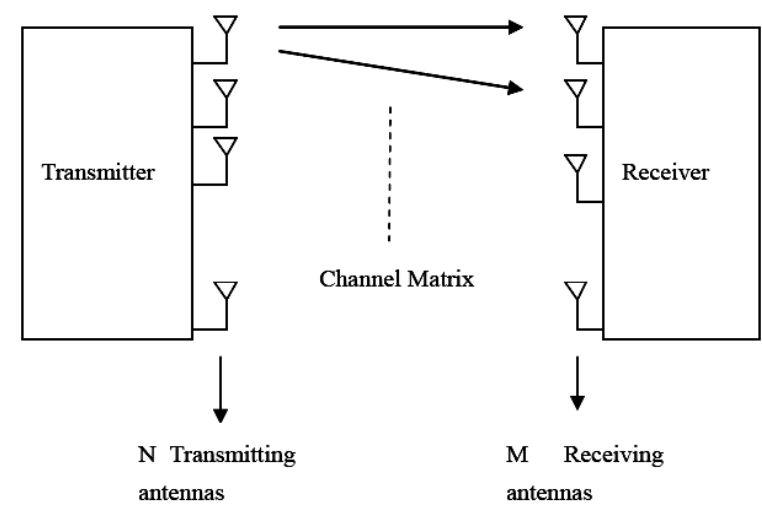

Fig-1: A MIMO Wireless Channel
Then in turn by selecting the best path, the signal quality can be improved to a greater extent thereby probability of error in the signal reception can be reduced dramatically[10].

MIMO employs diversity at both the transmitter and at the receiver side. The basic mechanism behind this technology is that the two different or similar data streams are transmitted through these spatially separated antennas and at the receiver side, using typical space time processing techniques i.e. alamouti and BLAST the streams are detected and received in more or less its original form.

Two representative space-time systems, namely space-time block coding (STBC) and vertical Bell Labs layered space time (V-BLAST), can improve system performance by providing diversity gain and multiplexing gain, respectively. [1] The classification chart of MIMO systems is shown in Fig.2. 


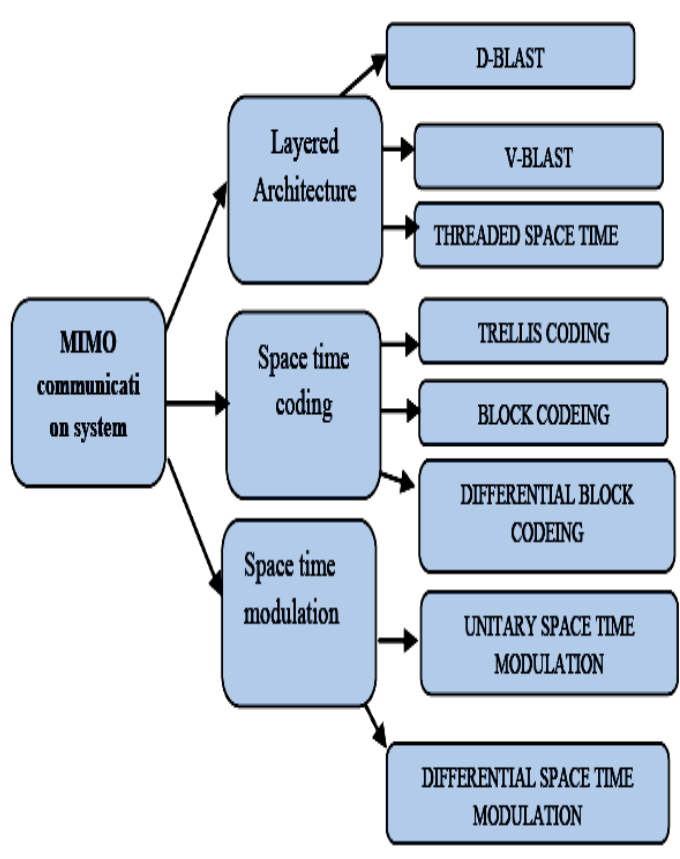

Fig-2: Summary Chart of MIMO systems

In this paper, the critical performance analysis of V-BLAST system has been discussed with MIMO so as to find the most effective diversity implementation for modern wireless systems. First part deals with the basic architecture of VBLAST system and the second part constitutes the modeling and simulation of V-BLAST structure along with the simulation results in image form.

\section{V-BLAST TECHNIQUE}

BLAST stands for Bell Laboratories Layered Space Time. As already discussed that the traditional approach of wireless communication system analyzes fading as a culprit because the images arrive at the receiver at slightly different times and can thus interfere destructively but with the use of antenna diversity techniques this multipath structure has become the boon for the betterment of system capacity. As contrary to the alamouti technique in which multiple antennas are transmitting the same signal at a time and at the receiver side, multiple antennas are receiving those many copies of data stream out of which best path will get selected, in this case the data stream is bifurcated into substreams proportional to the no. of transmitting antennas and then individual antenna will transmit each independent substream which all are going to received and detected by multiple receivers. The idea behind the technique is to increase the data rate of the system dramatically by taking the advantage of multiplexing gain property of antenna diversity.

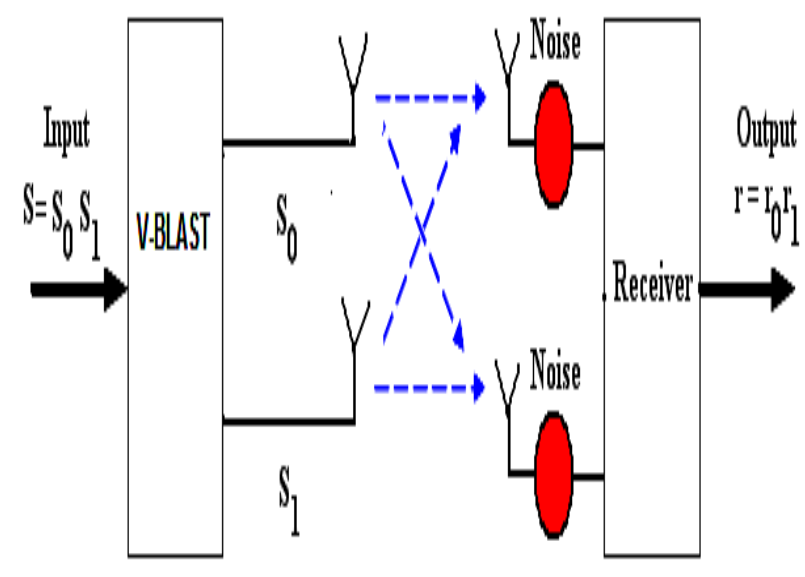

Fig-3: V-BLAST Architecture

Basically the two main techniques of BLAST are popular. One is Diagonal BLAST (D-BLAST) and the other is Vertical BLAST (V-BLAST). In case of D-BLAST, the ordering and decoding structure follow the diagonal pattern or circular pattern which will become more and more complex as the no. of transmitter and/or receiver antenna will increase. Hence to reduce the complexity, Fochini has introduced the simplest version of BLAST known as V-BLAST which has been thoroughly discussed in the forthcoming sections.

In V-BLAST a single data stream is split into multiple substreams and multiple transmitter antennas are used to simultaneously launch the parallel sub streams as shown in figure 3. All the sub-streams are transmitted by using the same frequency band, so spectrum efficiency will improve drastically. Moreover the user's data is being sent in parallel over multiple antennas, the effective data rate is increased in roughly in proportion to the number of transmit antennas used. One thing that has to be made sure is number of receivers for this system must be equal to or greater than that of the transmitter. The transmitted sub-streams are independent of one another. Individual transmitter power is scaled by $1 / \mathrm{M}$. So, total power remains constant independent of number of the transmitters (M).

At the receiver, again the multiple antennas i.e. array of antennas is used to receive the multiple transmitted data sub streams and their multipath replicas. Each receiver antenna receives all the data sub streams that are transmitted by no. of antennas. These all streams approaching to the individual receiver are not separate but are superimposed over one another. However, because of sufficient multipath scattering observed by different data substreams is different, by the use of quality signal processing algorithms the individual substreams will be detected from the multiple copies of it at each receiver side. In effect, the unavoidable multipath is exploited to provide a very useful spatial parallelism that is used to greatly improve data transmission rates. Thus, when 
using the V- BLAST technique, the more multipath, the better, just the opposite of conventional systems. Basically there are three main methods for signal detection in V-BLAST receiver. 1. Zero Forcing (ZF) Detection Algorithm

2. Minimum Mean Square Error (MMSE) Detection Algorithm

3. Maximum Likelihood (ML) Detection Algorithm

Here the approach to a lower complexity design of the receiver is to use a "divide-and-conquer" strategy instead of decoding all symbols jointly. First, the algorithm detects the strongest symbol. Now as one symbol is detected, its effect on the remaining symbols should be nullified so as to have less interference hence as a next step the strongest symbol will be subtracted from the replicas of the data stream. The algorithm continues by canceling the effects of the detected symbol and the decoding of the next strongest symbol until all symbols are detected. The optimal detection order is from the strongest symbol to the weakest one.

The Shannon's channel capacity for this system is given by [5],

$$
C_{V-B L A S T}=\log _{2} \operatorname{det}\left(I_{M}+\frac{P_{T}}{M \sigma^{2}} H^{T} H\right)(\text { bits } / \mathrm{sec} / H z)
$$

\section{MODELING \& SIMULATION OF V-BLAST TECHNIQUE IN MIMO ENVIRONMENT}

The very first MIMO architecture to achieve high data rate was proposed by G. J. Foschini which was known as the Belllabs layered space time architecture (BLAST). [4] VerticalBLAST is the finest algorithm that can increase the channel capacity to a extent that is defined by Shannon's channel capacity law under rich multipath scenario. In BLAST technique, multiple transmitting antennas transmit different data streams in parallel fashion which are spatially multiplexed. These data sub streams are transmitted simultaneously within the same bandwidth. With rich multipath scattering environment, these different streams are detected separately on receiver side based on the amount of fading experienced by all.

V-BLAST architecture is designed by using MT spacially separated antennas that are transmitting corresponding data substreams and NR receiving antennas to receive all the replicas of data sub streams. After encoding process each substream is fed to an individual transmitter. The type of modulation method utilized in these systems usually is $\mathrm{M}$ Quadrature Amplitude Modulation (MQAM). QAM combines phase modulation with amplitude modulation, limiting the channel bandwidth and making it an efficient method for transmission of data. BLAST's receivers operate over all data substreams transmitted by MT transmitters and apply various signal processing algorithms to detect the individual sub streams. For efficient operation of the detection algorithms, it is usually required that NR $>$ MT.

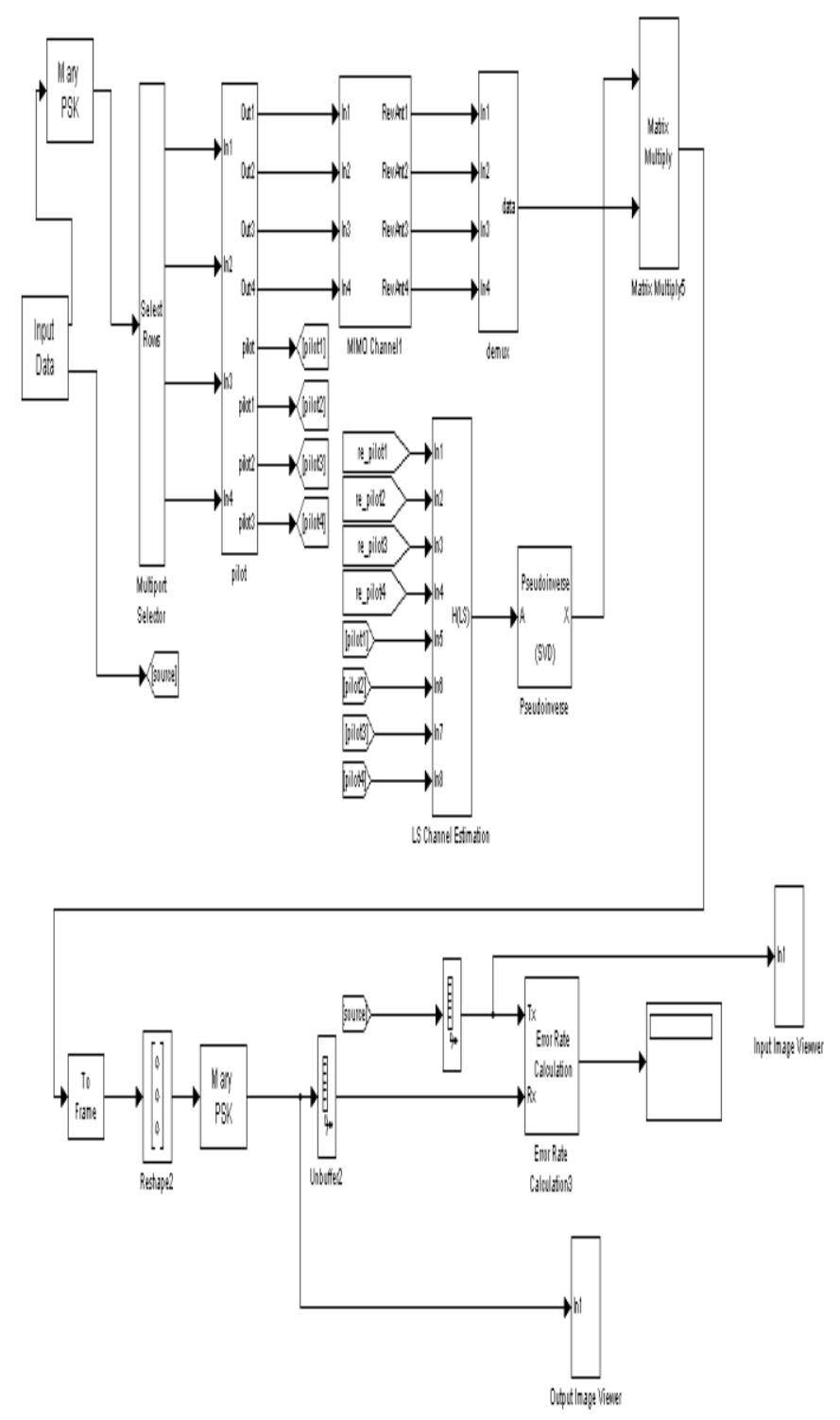

Fig-4: MIMO-VBLAST Model

As per the snap shot, the model is designed for $4 \times 4$ antenna system. Here the incoming data stream is bifurcated into four sub streams and is transmitted through four AWGN channels; all which are experiencing different amount of fading. This modulated data sub streams are mixed with some fixed size of pilots which are used to estimate the channel conditions. At the receiver side, these pilots are extracted and finally through ML detection algorithm, the data stream will be detected and received. At a final stage, demodulation and decoding is performed so as to recover the original signal back. 


\section{SIMULATION RESULTS \& DISCUSSION}

As per the snap shot of VBLAST model shown in figure-4, by setting the value of $\mathrm{SNR}=24 \mathrm{~dB}$ is simulated in MATLAB environment.

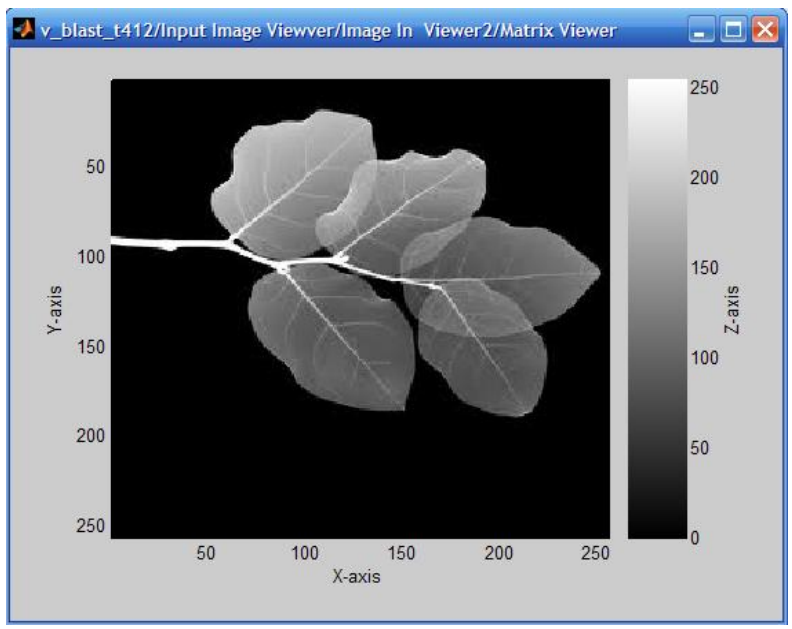

Fig-5(a): Input Image

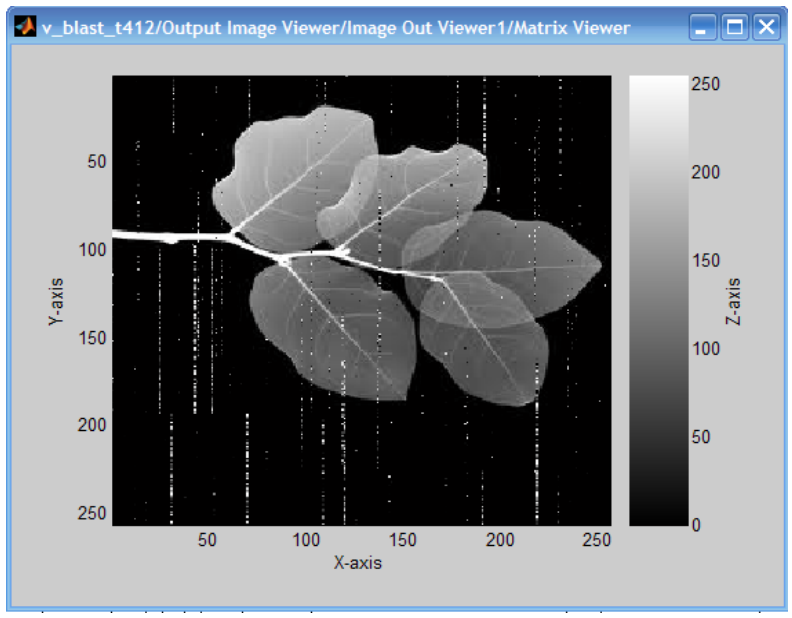

Fig-5(b): Output Image

Once the model is simulated, the performance will be evaluated by means of BER calculator measurement.

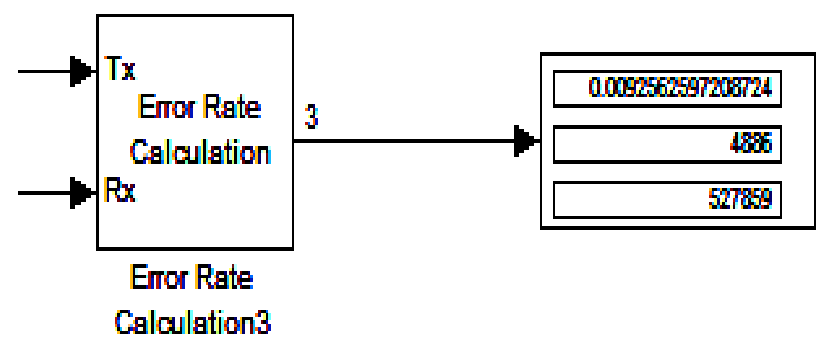

Fig-5(c): BER Calculator
By the transmission of almost same no. bits in terms of real time image signal, i.e. transmission of 527859 bits, 4886 bits get corrupted leading to the BER of around 0.009.

\section{CONCLUSIONS}

- Performs well when channel can be estimated properly.

- Degradation due to channel estimation errors is fairly high.

- Successive Interference Cancellation (SIC) makes for low complexity.

- Danger of error propagation that is inherent of a SIC scheme.

- Inferior to STBC due to lack of diversity gain at the transmitter.

\section{FUTURE WORK}

MIMO extremely promising but more validation work are needed. Currently MIMO with STBC is providing superb diversity gain but the data rate acquired is comparatively low, while with the implementation of MIMO-BLAST, though the data rate through multiplexing gain is high enough but the diversity gain at the transmitter side is nil. Plus the receiver complexity is also high with ML detection. So to combat with future demands, the hybrid MIMO i.e. combination of MIMOBLAST and MIMO-STBC would be the best solution. Further this can also be utilized in current wireless techniques so as to make them more efficient in terms of data rate or system capacity.

\section{REFERENCES}

[1]. A.L. F. de Almeida, W. C. Freitas Jr., J. C. M. Mota, F. R. P. Cavalcanti and R. L. de Lacerda Neto (2003), "Performance of MIMO Systems with a Hybrid of Transmit Diversity and Spatial Multiplexing", XX Symposiums, Brasileiro De Telecommunication, Rio De Janeiro.

[2]. Ahmed S. Ibrahim, Mohamed M. Khairy, and A. F. Hussein (2004), "Multi Layered Space Time Block Codes For OFDM Systems", Proc. The International Conference on Electrical, Electronic and Computer Engineering, Ain Shams University, Egypt.

[3]. Angela Doufexi, Andrew Nix, Mark Beach (2005), "Combined Spatial Multiplexing and STBC to Provide Throughput Enhancements to Next Generation WLANs", IEEE transaction.

[4]. Fenghua Li, "Performance Analysis of V-BLAST Detectors for the MIMO channel."

[5]. Nirmalendu Bikas Sinha, R. Bera, M. Mitra (2005), "Capacity and V-BLAST Techniques for MIMO Wireless Channel", Journal of Theoretical and Applied Information Technology.

[6]. Rui Zhang and John M. Cioffi (2008), "Approaching MIMO-OFDM Capacity With Zero-Forcing V-BLAST Decoding and Optimized Power, Rate, and Antenna-Mapping Feedback", IEEE Transactions on signal processing, vol.56, No. 10. 
[7]. W. C. Freitas Jr., A. L. F. de Almeida and F. R. P. Cavalcanti, "Interference Cancellation Receiver for Space Time Block Coded Systems Over Frequency Selective Channels", IEEE transaction.

[8]. Yu-Jin Song, Seong-Weon Ko, Hyun-Jin Park, Hun-Hee Lee, and Hyoung-Kyu Song (2010), "A Novel Efficient Detection Scheme for Hybrid STBC in MIMO-OFDM Systems", IEEE International Conference on Communication Technology, pp. 701-704, 978-1-4244-6868-3.

[9]. Chee Wei Tan and A. Robert Calderbank (2009), "Multiuser Detection of Alamouti Signals", IEEE TRANSACTIONS ON COMMUNICATIONS, VOL. 57, NO. 7.

[10]. Amitabh Kumar, "Mobile Broadcasting with WiMAX: Principles, Technology, and Applications", Focal Press Media Technology Professional Elsevier.

\section{BIOGRAPHIES}

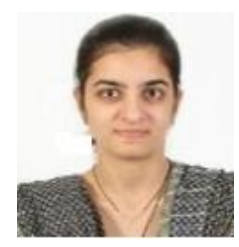

NIRALI A. KOTAK, She is a Ph.D. research scholar in JJT University Rajasthan in Electronics and Communication Department as well as working as an Asst. Prof. in Gandhinagar Institute of Technology, Gujarat.

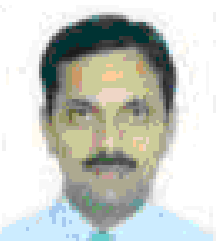

Dr. VED VYAS DWIVEDI, He is a Provice Chancellor at C.U.Shah University, Wadhwan, Gujarat. He has guided number of Ph.D. and PG research projects 